\title{
As-Is Analysis and Efficient 3D Layout Planning during the Conversion of Old and Design of New Production Facilities by Means of 3D-CAD
}

\author{
Björn Schuster \\ Business Development, N+P Informationssysteme GmbH \\ schuster@nupis.de
}

\begin{abstract}
Time is money in the area of production, especially when components or assemblies are to edit, assemble or to pack in large numbers. In many cases, an advanced automation is needed to provide everything necessary and to achieve throughput. New planning, simulation and visualization technologies improve the process of factory planning.
\end{abstract}

Keywords: Digital factory, Factory Design, 3D layout planning.

\section{Introduction}

The quality of factory planning today is an important factor to ensure the competitive position of factories. Global competition calls for the best possible planning and a permanent optimisation of the production processes, which is why the constant improvement of models, methods and tools for factory planning and operation is very important. The challenge facing many factory planners in connection with this development is to plan and implement the conversion of old and design of new production facilities as efficiently as possible.

\section{Digital Factory Planning Using 3D-CAD Software}

The majority of CAD data that is relevant for a factory today is available in 2D drawings and can only be transferred to a 3D format in stages and for specific projects on account of the effort involved and the generally existing departure from reality. There are a large number of software tools that allow virtual reality to be used to visualise the manufacturing planning of a factory. Virtual reality has become established in product development under the catchword CAD (Computer Aided Drafting) and is used in factory planning under the concept of a 'digital factory'. 'Digital factory' therefore means a virtual two- or three-dimensional representation of the factory to carry out real planning and a test-driven simulation, in particular to visualise a material flow analysis.

The use of 3D-CAD software accelerates the planning process since the planner can develop and communicate an efficient factory layout using a digital model. The 
subsequent simulation of a factory is understood as meaning the emulation of a factory system, with all of the associated dynamic processes, in an experimental model. The goal is to get as close as possible to reality so that know-how obtained from the simulation can be transferred to reality, in other words the real factory (see Bracht, Geckler, Wenzel 2011, p. 318).

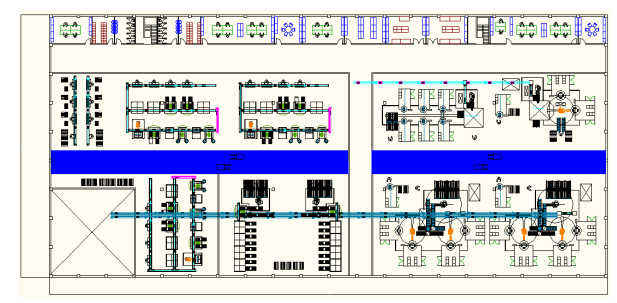

(a)

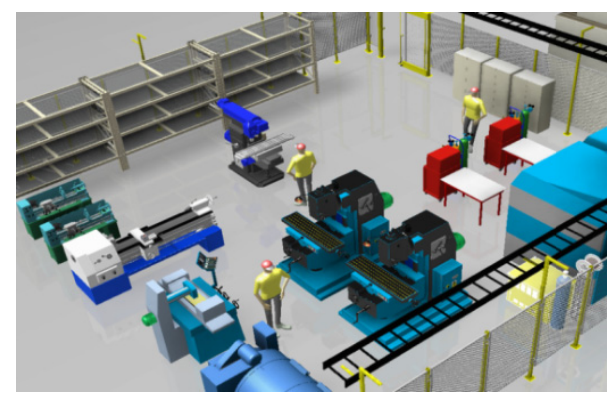

(b)

Fig. 1. From 2D layout planning to 3D layout planning with the Autodesk ${ }^{\circledR}$ Factory Design Suite (Autodesk 2012)

Autodesk ${ }^{\circledR}$ Factory Design Suite is a comprehensive software package that has been well received in the industry and which was written especially for the virtual development of layouts for production lines and complete production facilities (see Figure). This allows the complex process from planning the building, through the actual installation of the production facilities, right down to planning the progress of the building work and transport routes, to be simulated. The suite extends the operational procedures in AutoCAD $\AA$ and Autodesk $₫$ Inventor $\AA$ with interoperable procedures that are only available in the suite as well as factory-specific components and content. It therefore improves efficiency, accuracy and communication in factory planning projects as well as flexible reactions to altered business requirements.

The Autodesk® Factory Design Suite accelerates factory planning through extensive special commands and a specific environment for the rapid development of factory layouts. Any model can be dimensioned and edited as necessary with the aid of intelligent plant objects such as walls, doors and prop grids. By integrating scatter plot scans, the as-is condition of a plant (hall planning) can be recorded in 3D and then converted or adapted to the virtual environment. Complex conversion work or retrofits can hence be carried out on a 3D layout with the optimum efficiency, quality and flexibility. Analyses of material flows, machinery utilisation and energy consumption are possible in the early engineering and design phases. Non-value-creating costs that are incurred through superfluous transport routes within the factory can be determined on the basis of several simulation scenarios and optimised directly in the 3D layout.

What makes the suite special is that a variety of plant, building and machinery manufacturer data can be managed and compiled in one model. The functions for a digital check and evaluation of the equipment as well as for collision detection and distance analysis thus allow a custom-fit construction of the factory geometry before any extensive investments have been made (see Autodesk® 2012). 


\section{Outlook}

The example of suppliers for the automotive industry shows just how urgent it has become to address the topic of a 'digital factory'. The closer a manufacturer and supplier work together, the greater the pressure to resort to a comprehensive IT solution such as the Autodesk ${ }^{\circledR}$ Factory Design suite. Digital planning, 3D-CAD models, simulation, the compilation of data in a factory model and standardised data exchange are important requirements for this development. Tomorrow's 'digital factory' will also call for an even greater exchange of process data, a topic that is still new for many companies outside the core zone of automobile production, but which is already possible today with the Autodesk ${ }^{\circledR}$ Factory Design suite (see Autodesk ${ }^{\circledR}$ 2011).

The topic of the 'digital factory' as well as the associated AS-IS analysis and efficient 3D layout planning during the conversion of old and design of new production facilities by means of 3D-CAD will also have to be dealt with in different forms by small and medium-sized enterprises. $\mathrm{N}+\mathrm{P}$ links individual software products and offers an overall, customer-optimised IT solution for factory development and planning, thus creating added value, efficiency and a cutting edge for the customer.

\section{Conclusions}

Optimize your factory layout before it becomes real. Autodesk (B Factory Design Suite helps you to design and communicate the most efficient manufacturing layout by creating a digital factory model. Evaluate quickly multiple "what-if" scenarios to determine the best solution before any equipment is installed. Factory-specific visualization and analysis tools are helpful by increasing innovations, design efficiency and communication.

\section{References}

1. Autodesk ${ }^{\circ}$, Autodesk Factory Design Suite 2013: new orders through innovation, p. 14 (2012)

2. Autodesk®. Digital factory - a topics for SMEs? (2011), http://usa .autodesk. $\mathrm{com} / \mathrm{adsk} / \mathrm{servlet} / \mathrm{pc} / \mathrm{item}$ ? siteID=403786\&id=15963402 (August 28, 2013)

3. Bracht, U., Geckler, D., Wenzel, S.: Digital factory: methods and practical examples, pp. 318-320. Springer, Heidelberg (2011) 